\title{
La historiografía literaria en América Latina: crisis, debates y desafíos
}

\author{
Literary Historiography in Latin America: \\ Crisis, Debates and Challenges
}

\author{
VÍCTOR BARRERA ENDERLE \\ Universidad Autónoma de Nuevo León
}

\begin{abstract}
RESUMEN: La historia de la literatura, como forma de estudio, ordenamiento y cuestionamiento, es de origen reciente. Su desarrollo se ha visto entrelazado no solo con el devenir de los campos literarios, sino con los avatares de la geopolítica: el nacionalismo, el regionalismo y ahora la globalización, han sido factores importantes en su configuración y en su crisis presente. En América Latina, la historia literaria ha sido un desafío crítico permanente porque ha tendido que establecer sus propios criterios y metodologías, ante la constante tentación de adoptar periodizaciones y cortes temporales provenientes de otras literaturas. ¿Cómo construir una historia de la literatura en la actualidad, cuando las nociones de canon y de campo literarios han cambiado profusamente? En este ensayo me propongo estudiar estas circunstancias, describir sus crisis, y exponer sus desafíos.
\end{abstract}

ABSTRACT: The history of literature, as a way of study, planning and questioning, is recent. Its development has been intertwined not only with the evolution of literary fields, but with the vicissitudes of geopolitics: nationalism, regionalism and now globalization; all of them have been important factors in its configuration and its present crisis. In Latin America, literary history has been a permanent critical challenge that has tended to set their own criteria and methodologies, with the constant temptation to adopt periodization models and temporary cuts from other literatures. How to build a history of literature today, when the notions of literary canon and literary field have changed profusely? In this essay, I am ready to study and analyze the Latin American literary historiography, and, from there, establish their crisis, challenges and defiance.

PALABRAS CLAVE: historiografía literaria, crítica literaria, literatura latinoamericana, literatura regional y canon literario.

KEYWORDS: literary historiography, literary criticism, Latin American literature, regional literature and literary canon.

RECIBIDO: 23 de febrero de 2018 • ACEPTADO: 29 de abril de 2018 


\section{VÍCTOR BARRERA ENDERLE \\ Universidad Autónoma de Nuevo León}

\section{La historiografía literaria en América Latina: crisis, debates y desafíos}

\section{El debate por la autoridad en el conocimiento del pasado literario}

En 1931 se celebró en Budapest el Primer Congreso Internacional de Historia Literaria. ${ }^{1}$ El tema central de la reunión en la capital de Hungría fue la discusión en torno a las metodologías para el estudio histórico de la literatura. Dos grandes tendencias dominaban el ambiente: la que estaba a favor del método histórico y la que estaba en contra. Entre el primer grupo destacaban, al menos, cinco corrientes de análisis: la literatura comparada, la literatura general, el enfoque sociológico, el método geográfico y el modelo generacional. En la oposición sobresalían: el método psico-histórico (la llamada biografía de la obra), el estético, el crítico, el biológico, el formalismo ruso y la historia literaria artística, que, como su nombre lo indicaba, se desentendía de cualquier otro factor que no fuera inherente a la obra.

La gran pregunta que rondaba en aquellas discusiones fundacionales era si la historiografía era un género literario o científico. De ser lo primero, debía establecer sus propios criterios de base; de ser lo segundo, tendría que estar sujeta a la comprobación y verificación de su método

Es importante tener en cuenta que tres años después, en el Congreso de Escritores en Moscú, los estudios literarios en el orbe soviético se radicalizarían con la imposición del realismo social y el materialismo histórico. 
de clasificación y periodización. Algunos antecedentes de esta postura eran la filología histórica, desarrollada en el siglo XIX, a la par del surgimiento de los Estados nacionales (y fundamentada en la concepción hegeliana del desarrollo histórico), ${ }^{2}$ y las tendencias positivistas y evolucionistas de autores como Ferdinad Brunetiére y su Évolution des genres dans l'histoire de la littérature, de 1890. El crítico francés, por ejemplo, siguiendo las teorías evolutivas darwinistas, postulaba un desarrollo casi biológico de las creaciones literarias, dividiéndolas en géneros y especies. En otras palabras, en las páginas de su tratado encontrábamos la concepción positivista (y teleológica) de la historia a secas. Una relación cronológica de hechos verificables, cuya sucesión era, por lo tanto, indiscutible. Este tipo de sistematizaciones decimonónicas representaron, en buena medida, una reacción a la concepción romántica del quehacer literario, que fusionaba vida, lenguaje, obra y contexto social. Herder, Madame de Staël y Saint Beuve, a su modo, habían acentuado la condición individual (fuera personal o nacional) del artista y su creación: tales postulados permanecerían como los cimentos de la llamada defensa del "arte por el arte".

Whilhem Dilthey, haciendo eco de esas premisas, pugnaba por una historia, social y humana, de las artes para poder comprenderlas (y no solo explicarlas), destacando con ello, el carácter individual de las producciones estéticas. En un futuro cercano, Benedetto Croce haría lo propio, con su particular teoría estética y su peculiar división de géneros. Sin embargo, conforme los estudios literarios se fueron sistematizando en el siglo Xx, con el desarrollo de la lingüística (la publicación y la difusión de los cursos de lingüística general de Ferdinand de Saussure), del formalismo y, posteriormente, del estructuralismo, la tendencia se encaminó hacia el carácter científico de la escritura de la historia literaria.

Varios años después del Congreso de Budapest, Rene Wellek y Austin Warren, en su clásico libro, que todos, o casi todos, consultamos en las aulas de las carreras de Letras, Teoría literaria, de 1948, tes-

Georg Lukács, en La novela histórica, lo describe de la siguiente manera: "El nuevo espíritu de la historiografía, más visible en los importantes historiadores franceses de la Restauración, se centra precisamente en la cuestión de cómo aportar pruebas históricas para el hecho de que la moderna sociedad burguesa ha nacido de la lucha de clase que hizo verdaderos estragos a lo largo de toda la 'idílica Edad Media', y cuya última etapa decisiva había sido la Revolución francesa” (Lukács 1966: 26). 
timoniaban la hegemonía de dichas perspectivas científicas a la hora de estudiar e historiar al fenómeno literario. Una cosa era segura para ellos, la historia literaria no se podía escribir sin un basamento teórico y crítico: "En la práctica nunca se ha escrito ninguna historia literaria sin principios selectivos y sin hacer algún intento de caracterización y valoración" (Wellek y Warren 1953: 70).

Desde el ámbito de la academia hispánica, tenemos, por ejemplo, el testimonio de José Alsina, quien, al revisar la concepción literaria de los griegos, establecía que "[...] la tarea de la historia literaria debe desembocar en el estudio de una serie de factores que determinan o fusionan la obra literaria: el medio, el público, la formación intelectual del público y del autor, la condición del escritor en cada época, el análisis de la mentalidad colectiva, para culminar en la pregunta fundamental: ¿qué es la literatura?" (Alsina 1991: 32).

Incluso la corriente estructuralista, al mediar el siglo XX, postuló, a su modo, la necesidad de contar con un criterio para realizar la historia de la literatura. Sin ir más lejos, Roland Barthes ${ }^{3}$ consideraba que era inevitable que la historia literaria, a pesar de su inmanencia, adoptara enfoques provenientes de los estudios sociológicos. Dejo en este punto los debates en torno a la disciplina, pues me parece que con lo mencionado basta para forjar una idea de la relevancia de la historia literaria en el siglo Xx.

El problema que me gustaría plantear, sin embargo, va un poco más allá del recuento cronológico y del repertorio de esquemas expositivos. Tiene que ver con los criterios de base, con las estrategias desplegadas para proponer un modelo de periodización. Pero, principalmente, está vinculado con una problemática mayor, a saber: la literatura latinoamericana. Es imposible intentar siquiera una historia de la literatura sin tener algunos presupuestos de base (tal vez el principal sería: ¿qué se entenderá por literatura? No es el único, sin embargo). Ahora bien, al

En el prólogo a El grado cero de la escritura afirma: "Este orden sacro de los Signos escritos propone la Literatura como una institución y evidentemente tiende a abstraerla de la Historia, pues ningún cerco se funda sin una idea de perennidad; pero allí donde se la rechaza, la historia actúa más claramente; por lo que es posible formular una historia del lenguaje literario que no sea ni la historia de la lengua, ni la de los estilos, sino solamente la historia de los Signos de la Literatura, y se puede descontar que esta historia formal manifieste a su modo, que no es el menos claro, su unión con la historia profunda" (Barthes 1997: 12). 
trasladar estas inquisiciones al campo de nuestro interés, las dificultades se agigantan. No solo resulta complicado definir el quehacer literario en el espacio geográfico que comienza al sur del Río Bravo, sino que, al intentar hacerlo, nos colocamos inmediatamente ante otras dificultades de no menor peso; menciono algunas: la relación entre literatura y nación; la condición poscolonial de los países latinoamericanos (es decir: la permanente tensión entre sus expresiones verbales y culturales y el fallo de autoridad - y legitimación - proveniente de las antiguas capitales metropolitanas, entiéndase Madrid y Lisboa, que regulaban, a través de gramáticas, diccionarios, academias de la lengua e historias literarias, la ordenación del canon); y las diferencias regionales al interior de las naciones. Ya me ocuparé de cada una de ellas.

Uno de los problemas centrales de la historiografía moderna es el concerniente a la unidad: ¿es posible escribir la historia de un individuo, de un pueblo, de una nación, sin establecer conexiones con otros sujetos, otros pueblos y otras naciones? Ya en su introducción a su monumental Estudio de la Historia, Arnold J. Toynbee exponía:

Los historiadores generalmente ejemplifican, más que corrigen, las ideas de las comunidades dentro de las cuales viven y obran, y el desarrollo en los últimos siglos, y más particularmente en las últimas generaciones, del presuntamente autárquico Estado soberano nacional ha llevado a los historiadores a escoger las naciones como los campos normales del estudio histórico. Pero ninguna nación o Estado nacional de Europa puede presentar, aisladamente, una historia que se explique a sí misma (Toynbee 1985: 9).

Para el caso de la literatura de América Latina, la circunstancia es más compleja: se tienen que hacer cruces, comparaciones, contrastes; entender el quehacer literario como proceso, pero también como reflexión crítica constante.

\section{Genealogía de la historiografía literaria latinoamericana}

¿Cuándo surge en Latinoamérica el interés por registrar el desarrollo literario? Los primeros atisbos datan del periodo colonial. Tenemos los 
empeños de la élite criolla ilustrada por dar cuenta de la cultura propia ante la mirada reduccionista de los peninsulares. Menciono algunos ejemplos: Juan José de Eguiara y Eguren y su Bibliotheca Mexicana de 1755 (detonada por la inquina del deán de Alicante, Manuel Martí, quien, en su obra Epístolas, publicada unos años antes, en 1735, había negado la existencia de la vida intelectual en el "nuevo mundo"); los jesuitas expulsos (Juan Ignacio Molina, Manuel Lacunza, Francisco Javier Clavigero), que también sentaron sus reales al recrear el espacio natal perdido; y José Mariano Beristáin y Souza, con su Biblioteca Hispano-Americana Septentrional, publicada entre 1816 y 1821 . Estas empresas, no exentas de tribulaciones, estaban marcadas por la condición colonial, por la subordinación de saberes y por la sumisión del quehacer literario a las funciones públicas, tanto en el espectro de la monarquía como al interior de los territorios de la Iglesia.

La era republicana asumió el modelo herderiano para construir las historias patrias, basado en la lengua, la memoria y el pasado comunes. El debate entre Andrés Bello y José Victorino Lastarria, en la década de 1840 , sobre la forma de historiar el pasado, marcaría dos de las principales tendencias historiográficas de ese periodo: la basada en las fuentes y documentos (conocida como ad narrandum) y la que se soportaba en la interpretación, con su sesgo filosófico (llamada también ad probandum). El debate comenzó luego de la publicación, en 1847, del "Bosquejo histórico de la constitución del gobierno de Chile durante el primer periodo de la revolución", de Lastarria, con prólogo (que más que prólogo era una defensa de este modo de aprehender el pasado) de Jacinto Chacón. Partidario de la filosofía de la historia, el chileno planteaba una interpretación general de los hechos, desdeñando las fuentes y dando prioridad a las "leyes supremas" del devenir histórico. En rigor, la polémica venía desde antes: en 1844, el mismo Lastarria había presentado en la Universidad de Chile sus Investigaciones sobre la influencia social de la conquista y del sistema colonial de los españoles en Chile. Bello, reaccionó, siguiendo a Jean-Charles-Leonard Simonde de Sismondi (en particular su Tratado de la riqueza comercial), Augustin Thierry y otros historiadores y tratadistas, pugnando por un equilibrio y dando prioridad a las particularidades: 
Pero la filosofía general de la historia no puede conducirnos a la filosofía particular de la historia de un pueblo, en que concurren con las leyes esenciales de la humanidad gran número de agencias e influencias diversas que modifican la fisionomía de los varios pueblos cabalmente como las que concurren con las leyes de la naturaleza material modifican el aspecto de los varios países. ¿De qué hubiera servido toda la ciencia de los europeos para darles a conocer, sin la observación directa, la distribución de nuestros montes, valles y aguas, las formas de la vegetación chilena, las facciones del araucano o del pehuenche? De muy poco, sin duda. Pues tanto debemos decir de las leyes generales de la humanidad. Querer deducir de ellas la historia de un pueblo, sería como si el geómetra europeo, con el solo auxilio de los teoremas de Euclides, quisiera formar desde su gabinete el mapa de Chile (Bello 1957: 237-238).

Lastarria evocaría, años después, en sus Recuerdos literarios (dados a la imprenta en 1878), el choque de miradas sobre el pasado en estos términos:

El informe de la Comisión [que había premiado el ensayo de Lastarria, pero a la vez criticaba su falta de fuentes] era, en nuestro concepto, tanto un resultado de la opinión antigua del sabio rector de la Universidad [Bello] sobre la filosofía de la historia, cuanto un eco de la resistencia que la opinión ilustrada de la época oponía a toda innovación trascendental en el dominio de las especulaciones científicas y literarias (2001: 184).

Una positivista, la otra progresista, ambas invariablemente teleológicas. La literatura, sin embargo, ocupaba un lugar pragmático en el desarrollo de las naciones: era la vía para la creación de la identidad y la difusión del ideario político liberal.

El planteamiento especializado y la búsqueda de modelos esquemáticos no llegarían sino hasta segunda década del siglo XX, cuando en la mayoría de los países de la región se celebró el primer centenario de vida independiente: antologías, cátedras, congresos se llevaron a cabo entre los fastos del festejo. La cátedra de literatura argentina, que inauguró por esos años Ricardo Rojas en Buenos Aires, y las primeras lecciones de literatura mexicana, impartidas en la Escuela de Altos Estudios, de cuño ateneísta, son claros ejemplos de estas tendencias. 
Me concentro ahora en los primeros esfuerzos historiográficos hechos en América Latina, tras los proyectos modernizadores de nación de corte popular, realizados, como recién señalé, durante las primeras décadas del siglo Xx. Me refiero, en concreto, a los trabajos críticos de José Carlos Mariátegui y de Pedro Henríquez Ureña; los primeros, enfocados en las condiciones de producción nacionales; los segundos, en las manifestaciones culturales continentales. No hace falta repetir aquí la famosa queja del intelectual dominicano sobre la escritura de las historias literarias en nuestras naciones; tampoco es necesario repetir su insistencia en elaborar listados de obras y autores representativos. La secuencia entre el descontento y la promesa, es decir, entre la necesidad de reaccionar ante la imposición de valores impuestos y la voluntad de concretar una literatura propia le sirven para registrar las producciones literarias decimonónicas. Es, a su modo, respuesta y corrección a la Historia de la poesía hispano-americana, de Marcelino Menéndez y Pelayo (un estudio comenzado en 1892 y publicado, en dos tomos, entre 1911 y 1913). "Nuestra literatura se distingue de España, porque no puede menos de distinguirse, y eso lo sabe todo observador" (Henríquez Ureña 1960: 257). ${ }^{4}$

Mariátegui, en su discusión retroactiva con José de la Riva Agüero, establece uno de los más importantes modelos de periodización, y de paso destruye toda la edificación idealista del concepto de nación y su relación con el fenómeno literario. "En la historiografía literaria, el concepto de literatura nacional tampoco es demasiado concreto. No traduce una realidad mesurable e idéntica" (Mariátegui 2002: 235). La lectura del crítico peruano pone el acento en las particularidades de las formaciones culturales de la región:

Una teoría moderna - literaria, no sociológica - sobre el proceso normal de la literatura de un pueblo distingue en él tres periodos: un periodo colonial, un periodo cosmopolita, un periodo nacional. Durante el primer periodo un pueblo, literariamente, no es sino una colonia, una dependencia

$4 \quad$ La mayoría de los trabajos críticos posteriores, sobre todo después de la segunda mitad del siglo XX, tratarían de verificar esa hipótesis.

5 En 1905, Riva Agüero publicó el estudio Carácter de la literatura del Perú independiente. 
de otro. Durante el segundo periodo asimila simultáneamente elementos de diversas literaturas extranjeras. En el tercero alcanzan una expresión bien modulada en su propia personalidad y su propio sentimiento (Mariátegui 2002: 239).

Desafortunadamente, las propuestas críticas de periodización de Mariátegui y Henríquez Ureña no tuvieron escuela inmediata y fueron ignoradas o, peor aún, tergiversadas de manera abrupta e injertadas a la fuerza en el modelo generacional: entre las metodologías expuestas en el congreso de Budapest, fue esta la que más arraigo tuvo en América Latina, incluso, me atrevería a afirmar, que perduran en la actualidad residuos de ella.

En su modelo generacional, expuesto en el famoso ensayo de 1923: El tema de nuestro tiempo, Ortega y Gasset dividía el pensamiento de cada época en dos actitudes respecto a la tradición y al pasado inmediato. Una era de aceptación, la otra de beligerancia. Continuidad y ruptura. Esto dividía, a su vez, a los intelectuales en dos grupos: la mayoría conservadora (refugiada en la retaguardia), y la minoría vanguardista, que luchaba contra la corriente de la inercia. "Por medio de la historia intentamos la comprensión de las variaciones que sobrevienen al espíritu humano" (146-147), dice Ortega, e inmediatamente apunta hacia la búsqueda de la "sensación radical" que impera en cada época: "Esta que llamaremos 'sensibilidad vital' es el fenómeno primario en historia y lo primero que habríamos de definir para comprender una época" (146147). Más adelante explica:

las variaciones de la sensibilidad vital que son decisivas en la historia se presentan bajo la forma de generación. Una generación no es puñado de hombres egregios, ni simplemente una masa: es como un nuevo cuerpo social íntegro, con su minoría selecta y su muchedumbre, que ha sido lanzado sobre el ámbito de la existencia con una trayectoria vital determinada (Ortega y Gasset 1947: 146-147).

Para Ortega y Gasset, la generación era el concepto más importante en la historia; representaba, en sus palabras, el gozne sobre el cual esta realizaba sus movimientos más significativos. La afinidad principal que la nutría era su condición temporal (más allá de los pros y 
los contras, de los antagonismos o las aspiraciones de sus miembros): "Parejamente podemos imaginar a cada generación bajo la especie de un proyectil biológico lanzado al espacio en un instante preciso, con una violencia y una dirección determinadas. De una y otra participan tanto sus elementos más valiosos como los más vulgares" (146-147), así describía el filósofo español el concepto, y a continuación explicaba su desarrollo:

Es ello simplemente que las generaciones nacen unas de otras, de suerte que la nueva se encuentra ya con las formas que a la existencia ha dado la anterior. Para cada generación, vivir es, pues, una faena de dos dimensiones, una de las cuales consiste en recibir lo vivido -ideas, valoraciones, instituciones, etc. - por el antecedente; la otra, deja fluir su propia espontaneidad (Ortega y Gasset 1947: 148-149).

En su lectura, hay épocas acumulativas, y, en contraste, épocas eliminatorias y polémicas. Cada generación tiene su vocación propia, su misión histórica. Octavio Paz, un ferviente discípulo de Ortega, que supo usar sus conceptos en beneficio propio (la idea de las generaciones, le sirvió para marcar su diferencia), resumió su influencia en estos términos: "La razón de la enorme influencia que ejerció Ortega y Gasset sobre la vida intelectual de nuestros países está, sin duda, en esta concepción suya de las ideas y los conceptos como para qués y cómos. Dejaron de ser entidades fuera de nosotros y se convirtieron en dimensiones vitales" (Paz 1994: 297). Para terminar confesando: "Como tantos otros hispanoamericanos de mi edad, frecuenté sus libros con pasión durante mi adolescencia y mi primera juventud. Esas lecturas me marcaron y me formaron. Él guio mis primeros pasos y a él le debo algunas de mis primeras alegrías intelectuales" (Paz 1994: 299). No fue el único, todavía hoy hay quien postula una defensa a ultranza de la esencia de lo literario (basta asomarse a las páginas de las revistas y suplementos de mayor circulación en el continente y en España).

Durante buena parte del siglo Xx, las historias literarias basadas en el modelo generacional florecieron a lo largo y ancho de las naciones hispanoamericanas. Y ahí están, como testimonio, los libros y manuales de Luis Alberto Sánchez, de Enrique Anderson Imbert y de Arturo To- 
rres-Rioseco, por citar solo algunos ejemplos emblemáticos. Al mediar el siglo Xx, y conforme la tendencia hacia la especialización de las humanidades aumentaba junto con el incremento de las universidades, el modelo tomó revuelo. Trabajos como Esquema generacional de las letras hispanoamericanas (1963), del cubano Juan José Arrom, o La historia y crítica de la literatura hispanoamericana, del chileno Cedomil Goic, establecieron moldes invariables de registros de obras y autores, en cortes temporales de 15 y 30 años. No importaban ni el contexto ni los conflictos al interior de los campos literarios. La aplicación del modelo generacional podía funcionar incluso hacia el futuro, anunciando las promociones venideras de escritores.

Fue el brasileño Antonio Candido, en su Formación de la literatura brasileña de 1959, quien puso de nuevo el acento en la particularidad, en la especificidad que la literatura había cumplido en nuestras sociedades (retomando, así, la labor emprendida por Henríquez Ureña). Centrándose en los momentos decisivos y en los grupos de autores, Candido promovió una perspectiva crítica (y por lo tanto contrastiva) de la historia literaria, con base en el estudio de:

[...] um sistema de obras ligadas por denominadores comuns, que permitem reconhecer as notas dominantes duma fase. Estes denominadores são, além das características internas (língua, temas, imagens), certos elementos de naturaleza social e psíquica, embora literariamente organizados, que se manifestam historicamente a fazem da literatura aspecto orgánico da civilizacão. Entre eles se distinguem: a existência de um conjunto de productores literários, mais ou menos conscientes do seu papel; un conjunto de receptores, formando os diferentes tipos de público, sem os quais a obra não vive; um mecanismo transmissor (de modo geral, uma linguagem, traduzida em estilos), que liga uns a outros. O conjunto dos três elementos dá lugar a um tipo de comunicacão inter-humana, a literatura, que aparece sob este ángulo como sistema simbólico, por medio do qual as veleidades, mais profundas do individuo se transformam em elementos de contacto entre os homens, e de interpretacão das diferentes esferas da realidade (Cândido 2000: 23). ${ }^{6}$

6 " $[\ldots]$ un sistema de obras ligadas por denominadores comunes, que permiten reconocer las notas dominantes de una fase. Estos denominadores son, además de las características internas (lengua, temas, imágenes), ciertos elementos de naturaleza social y psíquica, aunque literariamente organizados, que se manifiestan históri- 
Las décadas de los sesenta y los setenta marcaron profundos cambios en los estudios literarios, si bien el enfoque generacional y el estructuralismo se mantenían (y, posteriormente, cobrarían fuerza con la instalación de las dictaduras militares en buena parte del Cono Sur), comenzaron a ensayarse nuevas formas de periodizar el pasado literario. Partiendo de la relectura del marxismo occidental, recientemente traducido al español (Lukács, Golmann, Benjamin y Adorno, entre otros), de la teoría de la dependencia, y del interés mundial despertado por el boom narrativo de los años sesentas, la crítica latinoamericana se enfocó en la descripción de las principales problemáticas que caracterizaban al campo literario y lo diferenciaban de las producciones artísticas y culturales provenientes de otras latitudes.

\section{La historiografía literaria hoy: problemas y desafíos}

Hablar de historiografía literaria en la actualidad (refiriéndonos en concreto a la literatura latinoamericana) presenta una serie de dificultades de gran calada; incluso para muchos sería un despropósito: asunto anacrónico del siglo pasado. En pocas palabras, algo obsoleto. Antes de rebatir tales afirmaciones, comenzaré por apuntar las evidentes dificultades. Primero, tenemos la crisis en las formas de representación y producción de conocimiento (crisis agravada desde las últimas tres décadas del siglo Xx con el llamado fin de los metarrelatos, entre ellos la manera de registrar el pasado y la forma de narrarlo); luego, el cuestionamiento de los conceptos de nación e identidad; y para seguir con la suma, añadamos los drásticos cambios en la misma noción de lo literario: desde su expansión a otros registros y soportes, hasta su muchas

camente y hacen de la literatura un aspecto orgánico de la civilización. Entre ellos se distinguen: la existencia de un conjunto de productores literarios, más o menos conscientes de su papel; un conjunto de receptores, formando los diferentes tipos de público, sin los cuales la obra no vive; un mecanismo transmisor (en general, un lenguaje, traducido en estilos), que se liga entre sí. El conjunto de los tres elementos da lugar a un tipo de comunicación interhumana: la literatura, que aparece bajo este ángulo como sistema simbólico, por medio del cual las veleidades más profundas del individuo se transforman en elementos de contacto entre los hombres, y de interpretación de las diferentes esferas de la realidad." 
veces declarada extinción. ¿Desde dónde hablar del pasado literario? ¿Qué criterio utilizar al hablar de obras y autores? ¿Cuál será nuestro concepto de nación, y cuál el de región? La formulación de preguntas podría seguir indefinidamente.

Como recién sugerí, desde finales de los años sesentas (el centenario del natalicio de Rubén Darío marcó un hito en la revisión crítica de las letras latinoamericanas) se han desplegado conceptos y enfoques que intentan dar cuenta de las particularidades. Nombro algunos de los más destacados: la transculturación narrativa, de Ángel Rama; la heterogeneidad cultural, de Antonio Cornejo Polar; la hibridación, de Néstor García Canclini; las sociedades abigarradas, del guatemalteco René Zavaleta. Además, los cuestionamientos a la construcción del canon por parte de críticos como Walter Mignolo; de las puntuales inquisiciones de las teorías feministas y de género; de los aportes y críticas desde y por las llamadas literaturas indígenas. Todos estos factores han contribuido al cuestionamiento de la disciplina, pero no han agotado ni clausurado su existencia, al menos no de manera definitiva.

¿Por qué? Para mí, la historia literaria latinoamericana, en todos sus procesos y registros, es la manifestación de la puesta en escena de diversos procedimientos críticos y apropiaciones teóricas en aras no solo del cuestionamiento o encumbramiento del canon, sino, y esto es lo fundamental, de la búsqueda por establecer una reflexión (implícita y explícita) sobre la literatura y sus múltiples formas de aprehenderla (contenidas aquí todas las formas de mediación experimentadas hasta ahora). Supongo que la historia que me interesa contar, en el fondo, es la de la crítica. De ahí que me cueste aceptar la historia literaria como algo obsoleto. Me parece, más bien, que las transformaciones actuales hacen más difícil cualquier intento de representación (y esa sensación de rechazo o sospecha permanecerá entre nosotros por un buen rato); esto, sin embargo, no conlleva la clausura de la empresa. El tiempo pasa, las obras siguen apareciendo y se registran de alguna u otra manera: si dejamos de lado la escritura de la historia literaria desde una perspectiva crítica y cultural, el mercado o las instituciones culturales, o los booktubers, se encargarán de hacerla o de inventarla según sus propios intereses y demandas. No satanizo ninguna de estas instancias o agentes (de hecho, los considero elementos a estudiar y a tomar en cuenta), solo 
señalo la existencia de los diversos registros historiográficos que conviven en el presente. Nuestras limitaciones (sean ideológicas, culturales, estéticas, políticas, académicas) no deberían convertirse en impedimento para arriesgar juicios, incluidos los de valor.

El proceso actual de la literatura (en sus múltiples variaciones, incluida aquí la llamada "posliteratura") debe o debería ser historizado de manera crítica, lo cual implicaría el distanciamiento temporal. No propongo, por cierto, algún tipo de objetividad o metodología rígida, aunque sí apelo al uso, cuando sea conveniente, de distintos recursos materiales, tal como sugiere Franco Moretti en su ensayo La literatura vista desde lejos: "[...] la cuantificación del análisis literario puede asumir formas diversas, desde la historia del libro hasta la estilística computacional, desde los bancos de datos temáticos hasta el análisis multivariado del léxico, entre otras aplicaciones" (Moretti 2007: 17).

Hay preguntas que me sigo formulando: ¿dónde quedaría aquí la noción de valor? ¿Es posible desentendernos de ella sin caer en la rigidez de lo "políticamente correcto"? Creo que todos estamos más o menos de acuerdo en que es imposible seguir sosteniendo cualquier tipo de esencialiación de la literatura; somos testigos de las diversas conceptualizaciones del quehacer creativo y de la multiplicidad de registros y soportes de los que hacen uso autores y autoras de todo tipo. Lo cierto, sin embargo, es que, sea desde el mercado o desde las mismas instituciones culturales latinoamericanas, se sigue imponiendo una noción unilateral de lo literario, que pretende borrar todo elemento contextual y coyuntural de su producción.

Me interesa, finalmente, indagar en la posibilidad de articular una historia literaria desde las regiones, es decir, desde los espacios no centrales de las naciones latinoamericanas. Mi propósito dista mucho de caer en las simples reivindicaciones de los regionalismos tradicionales. El elemento regional, llamémosle así provisionalmente, sería una forma de complemento y cuestionamiento de la elaboración de los cánones nacionalistas: serviría para contrastar las formaciones discursivas hegemónicas, evidenciando la complejidad y heterogeneidad de los campos literarios. Tal como apuntaron en su momento Mariátegui y Antonio Cornejo Polar. Partiría, para ello, de algunos cuestionamientos: ¿el campo literario regional está determinado por características 
peculiares? O, a la inversa, ¿su conformación se encuentra en completa dependencia de elementos externos, impuestos verticalmente desde espacios hegemónicos, en los que predomina el capital simbólico? Para ahondar en estas inquisiciones sería necesario echar mano de las nuevas perspectivas de la historia regional latinoamericana, ${ }^{7}$ que ha establecido dos enfoques o esquemas básicos para el estudio histórico de estos espacios: el dendrítico y el solar:

En el primero, la articulación del espacio regional se da en función de la dependencia a centros o espacios metropolitanos (sean nacionales o supranacionales), desde los cuales se controla la economía, la política y, añadimos, la cultura; y se impide (o se regula) el diálogo entre regiones y el desarrollo autónomo. En el segundo, el acento se pone en la diferencia, en las particularidades del lugar, respondiendo a variables y flujos internos. Llevados al extremo, cada uno de estos esquemas configuraría una visión unilateral, ya sea desde la total sumisión o subordinación, o desde un chovinista deseo de individualización que estropearía cualquier tipo de acercamiento crítico (Barrera Enderle 2014: 9).

El desafío es, pues, múltiple, pero no por ello imposible: la historiografía literaria regional sería una herramienta más, en un amplio espectro de posibilidades críticas enfocadas a abordar la literatura latinoamericana reciente. Formas de acercamiento que, sin descuidar las particularidades, nos permitieran ver el conjunto y entender el proceso. Tampoco descarto la posibilidad de utilizarla tanto en la academia (como forma de enseñanza, pero también, y principalmente, como vía para la discusión y la reflexión), como en la crítica pública (una manera de contrarrestar la hegemonía del mercado).

El registro histórico de la literatura latinoamericana actual podría ser uno de los ejercicios críticos más interesantes a la hora de dar cuenta del pasado inmediato: se podrían desbaratar mitos, revelar campañas publicitarias (disfrazadas de "sesudos juicios críticos"), y encontrar redes comunicativas insospechadas. Las dificultades, que no son pocas, formarían parte del proyecto. Las enumero brevemente: el peligro de caer

Véase el ensayo de Pedro Pérez Herrero (1991): Historia y región en México (1700-1850). 
en las generalizaciones; el siempre complicado acceso a los materiales; la necesidad permanente de crear nuevos instrumentos y dispositivos, tantos críticos como teóricos; el enfrentamiento (o confrontamiento) con las políticas, burocráticas y administrativas, de los comités de ciencias y tecnologías, y la imposición de sus metodologías rígidas.

Después de casi cien años de reflexiones sobre las condiciones (materiales y simbólicas) de la historia literaria latinoamericana, aún seguimos debatiéndonos, tal como lo expuso en su momento Pedro Henríquez Ureña, entre el desencanto y la promesa, entre las dificultades existentes y las posibilidades de exploración. Hoy, como entonces, la necesidad de crear grupos de autores y establecer momentos decisivos (para luego discutir la validez o la falta de validez de su inclusión o exclusión) sigue vigente. Todavía hay mucho trabajo por hacer. En la pregunta lanzada en Budapest, en 1931: ¿es la historiografía literaria un género de la literatura o es una expresión del discurso científico? (en otras palabras: escoger entre una exploración crítica o una simple metodología cuantitativa), nos hemos inclinado por la primera opción: ha llegado el momento de demostrarlo.

\section{Bibliografía}

Alsina, José (1991). La teoría literaria griega. Madrid, Gredos.

BARRERA ENDERLE, Víctor (2014). Siete ensayos sobre literatura y región. Monterrey/México, Facultad de Filosofía y Letras/Universidad Autónoma de Nuevo León.

BARTHES, Roland (1997). El grado cero de la escritura. Traducción de Nicolás Rosa. México, Siglo XXI Editores.

Bello, Andrés (1957). "Modo de escribir la historia", en Obras completas, vol. XIX. Temas de historia y geografía. Caracas, Ministerio de Educación/ Comisión Editora de las Obras completas de Andrés Bello/Biblioteca Nacional.

CÂNDIDO, Antonio (2000). Formação da literatura Brasileira. Belo Horizonte-Río de Janeiro, Itatiaia Limitada.

Henríquez UReÑa, Pedro (1960). "Seis ensayos en busca de nuestra expresión”, en Obra crítica. Edición de Emma Susana Speratti y prólogo de Jorge Luis Borges. México-Buenos Aires, Fondo de Cultura Económica. 
LASTARRIA, José Victorino (2001). Recuerdos literarios. Prólogo de Luis Sánchez Latorre. Santiago, LOM Ediciones.

LuKÁcs, Georg (1966). La novela histórica. Traducción de Jasmín Reuter. México, Era.

MARIÁTEGUI, José Carlos (2002). “El proceso de la literatura”, en Siete ensayos de interpretación de la realidad peruana. Lima, Amauta.

MoretTi, Franco (2007). La literatura vista de lejos. Traducción de Marta Pino Moreno. Barcelona, Marbot Ediciones.

Ortega y Gasset, José (1947). El tema de nuestro tiempo en Obras completas, vol. III. Madrid, Revista de Occidente.

PAZ, Octavio (1994). "El cómo y el para qué: José Ortega y Gasset", en Fundación y disidencia. Dominio hispánico, Obras completas, vol. 3. México, Fondo de Cultura Económica.

Pérez Herrero, Pedro (1991). Historia y región en México (1700-1850). México, Instituto Mora/Universidad Autónoma Metropolitana.

ToynbeE, Arnold J. (1985). Estudio de la historia. Traducción de Luis Grasset. Vol. I. México, Editorial Planeta.

Wellek, Rene y Austin WarRen (1953). Teoría literaria. Traducción de José María Gimeno Capella. Madrid, Gredos. 